\title{
ERratum
}

\section{Progress in physical properties of Chinese stock markets}

\author{
Yuan Liang (梁源 $)^{1,2}$, Guang Yang (杨光 $)^{1}$, Ji-Ping Huang (黄吉平 $)^{1, \dagger}$ \\ ${ }^{1}$ Department of Physics and State Key Laboratory of Surface Physics, Fudan University, Shanghai 200433, China \\ ${ }^{2}$ Department of Applied Physics, College of Science, Donghua University, Shanghai 201620, China \\ E-mail: ${ }^{\dagger} j p h u a n g @ f u d a n . e d u . c n$ \\ Received September 8, 2013
}

Erratum to: Front. Phys., 2013, 8(4): 438

DOI 10.1007/s11467-013-0366-0

Due to typesetting errors, some labels were missed in Fig. 5(a). The corrected figure should be

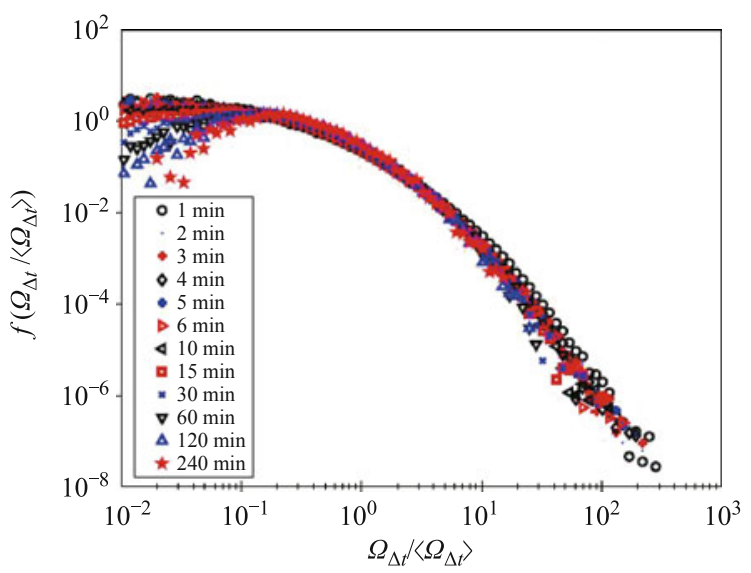

The online version of the original article can be found at

http://dx.doi.org/10.1007/s11467-013-0366-0 